\title{
TRAFFIC SIGN DETECTION USING DEEP LEARNING
}

\author{
Anushka Chauhan, Aman Rastogi, Agrima Gaur, Anugrah Singh \\ Department of CSE \\ IMS Engineering College, Ghaziabad, U.P., India
}

\author{
Mrs. Shaili Gupta \\ Department of CSE \\ IMS Engineering College, Ghaziabad, U.P., India
}

\begin{abstract}
Convolutional Neural Networks mostly use deep learning algorithms to detect and identify traffic signs till now but they are lacking in so many ways. This paper will give a really effective method for traffic sign detection and identification using convolutional neural networks. Convolutional Neural Networks are used for road sign detection and classification as it takes an input image and then assigns weights to different aspects in the image and then differentiate them from each other. Other classification algorithms require much longer preprocessing than the ConvNet. The filters which are there in primitive methods are engineered manually with training. These filters are learned by the ConvNets. Neurons respond to stimuli in the receptive field only, which is a restricted region of the visual field. The temporal and spatial dependencies of an image can be successfully captured by applying the relevant filters. To understand the sophistication of an image in a better way, the network can be trained. After reducing the parameters and weights reusability, the architecture fits better with the image dataset. The architecture of the system is designed in such a way that it extracts important features from the traffic sign's images and classifies them under various categories.
\end{abstract}

\section{Keywords - Dataset, Road Sign, ConvNet, Deep learning.}

\section{INTRODUCTION}

Traffic sign detection is extremely important for road safety and it has plenty of complications. Even a minor confusion in detecting a traffic sign may result in severe consequences involving the loss of lives of groups of people. Traffic signs are very useful as they supply a lot of data about the state of the road, warnings that are important for traveling on roads safely. Failure of noticing these signs may result in fatal road accidents. In such conditions, if we could make a mechanized road sign detection and identification system, then it might be very helpful for the drivers if they may get distracted from their driving. The camera is placed to capture the time images and they are fed to the model. The vehicles are running on the road in different conditions like low light conditions, rainy conditions, and foggy conditions, etc that the deep learning algorithms should be reliable in each of such conditions. The perfect deep learning algorithms to capture these traffic signs and with different orientations and for recognizing them we are using convolutional neural networks.

As their performance is so much affected by the prevailing environmental conditions and also various other temporary and permanent situations can affect the perceptibility of road signs. The traffic sign detection and recognition system face difficulties like non-identical traffic signs and bad postage of signs. This paper gives a detailed description of the issues of road sign detection and the solution to many of these problems. CLAHE (Contrast Limited Adaptive Histogram Equalization) has been used in improving the contrast of different traffic sign images. CLAHE is an algorithm whose implementation can be found in the Scikit-Image Library.

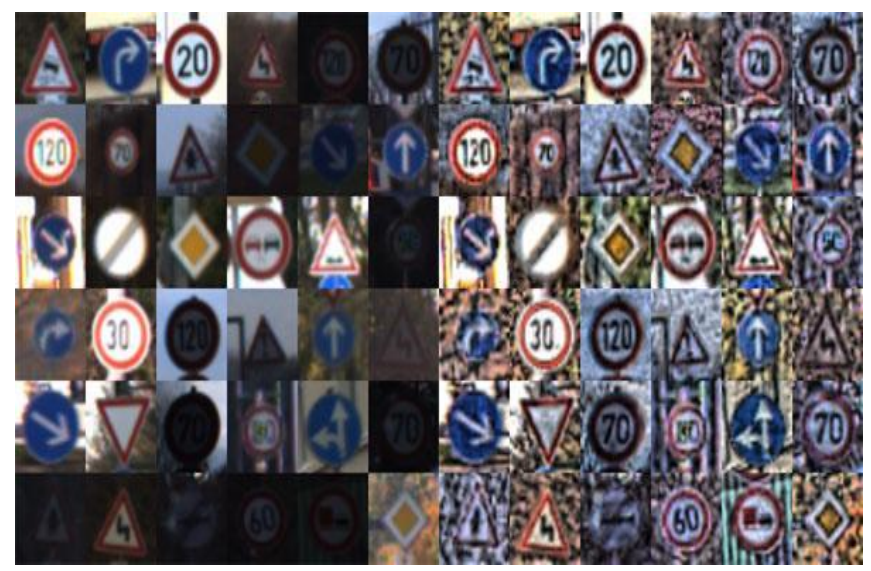

Fig. 1.Original Images are present on the left side.After applying CLAHE, image contrast is improved and the images are seen on the right side

In the tasks associated with images, the deep learning neural network methods are used. The kernel function in the convolutional layer captures the image and its spatial information. A convolutional neural network consists of layers 
and these layers are input layer, hidden layers, and output layer. There can be one or more than one hidden layer. Convolutional pooling, fully connected layers, and normalization layers are present in the form of hidden layers. CNN performs very effectively within the operations associated with images but it has some drawbacks also. Change in a pose or image orientation can result in confusions in $\mathrm{CNN}$. To reduce the spatial information associated with data and for downsampling of information, a max-pooling layer is employed within the convolutional neural network.

\section{PROPOSED WORK}

Development of self driving cars and intelligent transportation systems have shown a growth evolution in the last decade. Road sign detection and identification is the most challenging task confronted by the researchers and developers. Road sign detection includes two steps. First is the feature extraction and second is the signs recognition. In the first step, many methods had been proposed like scale invariance feature, edge detection, histogram of gradient and others. Many algorithms are used like SVM, KNN classifier, artificial neural network etc. Currently traditional computer vision algorithms are gradually replaced by the different applications like object classification and pattern recognition.

\section{A. Dataset}

For developing a traffic sign detection and identification system, the essential requirement is a traffic sign database. A huge number of traffic sign photos must be present in the traffic sign database. To train the system, a rich dataset with various types of images is required in object recognition. German Traffic Sign Recognition Benchmark(GTSRB) is used for the purpose of traffic sign detection and identification. Description and visualization of the GTSRB dataset was done. After driving for 10 hours on different roads of Germany this dataset was created and this dataset is publicly available. The dataset was reduced to 43 classes and around 50,000 images after removing the repetitive and redundant frames. The size of the images in the dataset is $32 \times 32$. Training data and testing data are two parts in which the dataset is divided. Training data is used in the training of the model. The final Neural Network can be evaluated by the testing data.

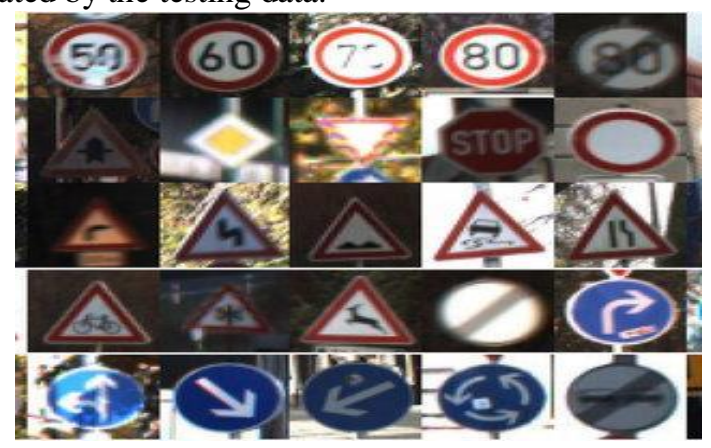

Fig. 2. GTSRB Data Set

\section{B. Neural Network Architecture}

A large number of neurons and layers are required in a fully connected neural network for image classification. Due to this thing, the number of parameters increases which leads to overfitting. The pixel correlation properties of the input image may be lost as all the neurons are connected with each other. This problem is solved by the convolutional neural networks with the help of their kernel filters. Keras Sequential API is used to build the convolutional neural network. A build method is designed which will take four parameters -width, height, depth and the classes. A set of layers is defined which is having convolutional, relu, batch normalization and pool layers. For distinguishing between different traffic sign colors and shapes, $5 * 5 \mathrm{kernel}$ is used in layers. Then two sets of CONV=>RELU layers are defined which will make the pool layers of our neural network architecture. Before max-pooling two sets of $\mathrm{CONV}=>\mathrm{RELU}=>\mathrm{BN}$ is applied so as to reduce the volume dimensionality. To prevent overfitting, dropout is applied.

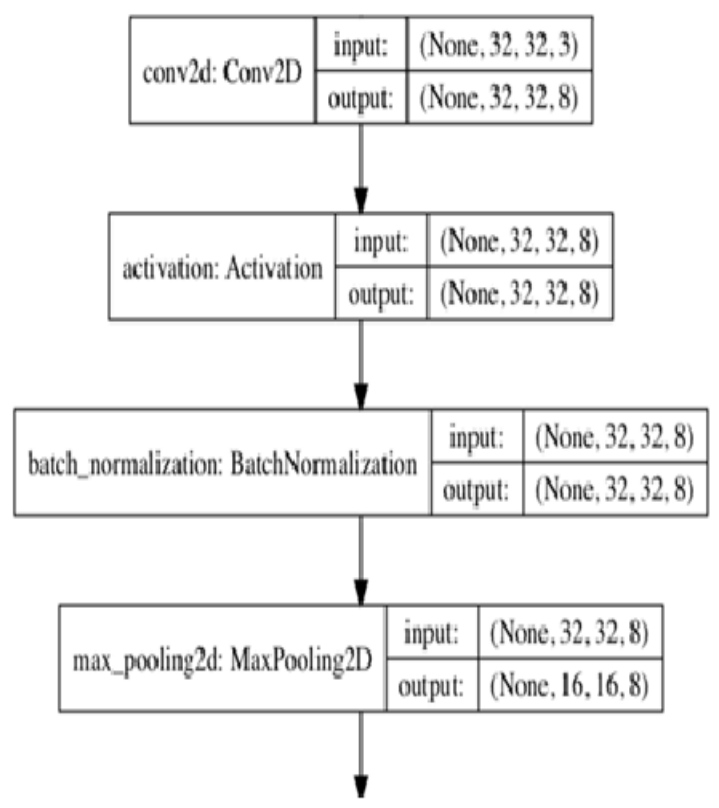

Fig. 3. Neural Network Architecture

\section{Deep Learning}

Deep learning implementations have replaced the previous methods that were used in recent years with increased computation power, available standard datasets, and access to a large amount of data. The highest accuracy rates are achieved by the convolutional neural networks, state of algorithms. A multi-stage neural network is called a convolutional neural network that is capable of learning all the features on their own. Every stage consists of a convolution layer, a nonlinear layer, and a pooling layer. For training each filter, a gradient descent optimizer has been used for minimizing the loss function. A 
classifier is fed with all the output from the layers which improves the accuracy of the classification.

\section{Machine Learning}

Traffic sign classification can be done by various types of machines like linear discriminant analysis, support vector machines, random forests, ensemble classifiers. Common covariance matrix and multivariate Gaussian present in class densities. The n-Dimensional data plane is divided with a hyperplane and this classification algorithm is called Support Vector Machines. Machine learning approaches were very time consuming and error-prone processes as they were not able to handle the variable size images and difference in the aspect ratios.

\section{EXPERIMENT AND RESULT}

A convolutional neural network is built and trained using the TensorFlow deep learning library [12]. A traffic sign classifier is trained to obtain over $95 \%$ accuracy using Keras and other deep learning libraries. The model successfully classifies the 43 traffic signs classes present in the dataset. The accuracy of the test data is evaluated and how to make predictions on new input data could also be learned through this. Through traffic sign classification, traffic signs present on the road like speed limit signs, merge signs, etc can be recognized.

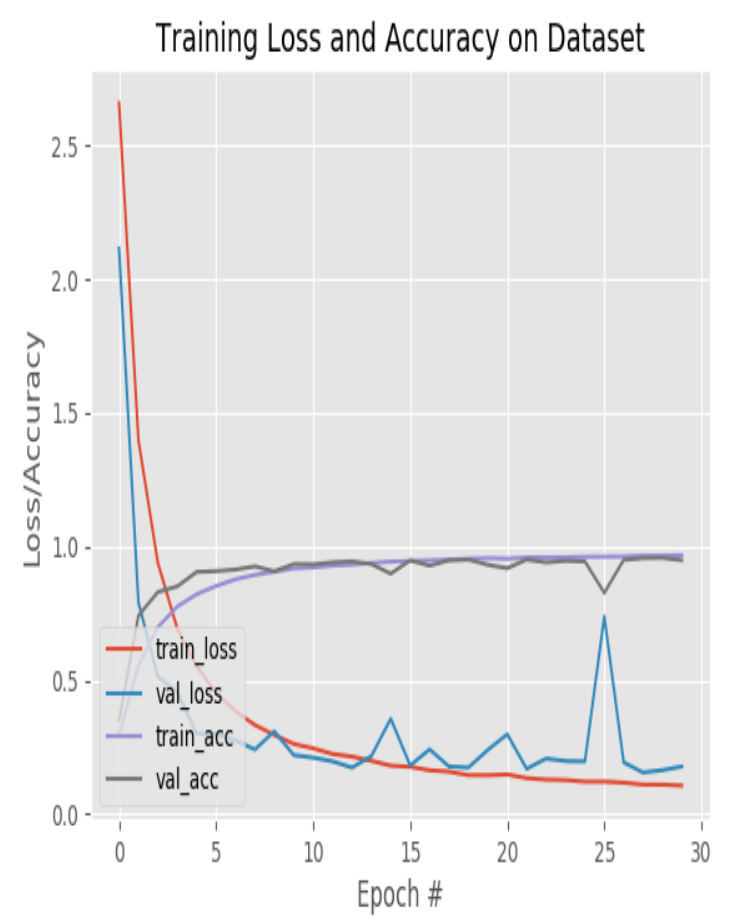

Fig. 4. Training Loss and Accuracy

The above graph shows that the accuracy keeps on increasing with the epochs and after 15 epochs it almost remains constant up to 25 th epochs. It then surges little and then again becomes constant. The training loss is gradually decreased. Some fluctuation is due to the fact that dropout is applied in the fully connected layers.

A sequential model is used and the set of layers is using a $5^{*} 5$ Kernel which is helpful for distinguishing between the different color blobs and the shapes of different traffic signs. The result is a more serializable model. The test images are processed with the model and a prediction of results is shown below.
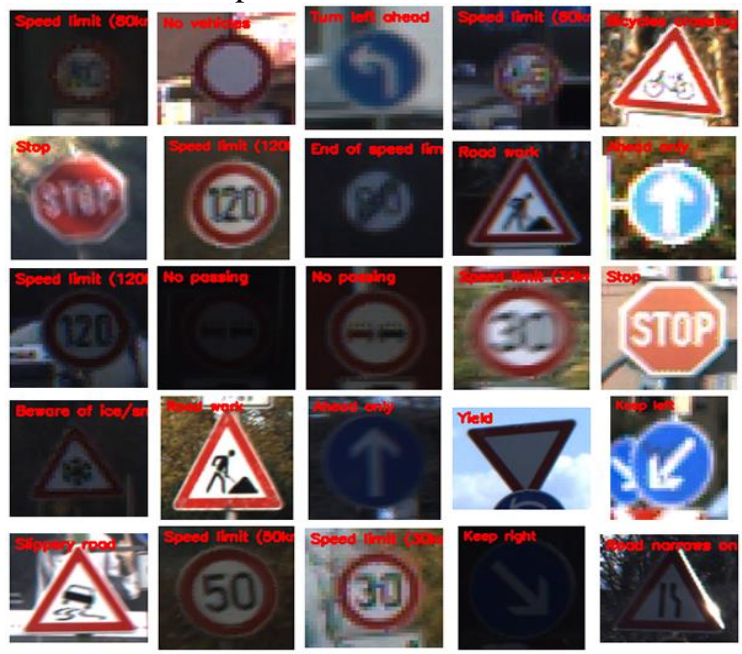

Fig. 5. Result of Traffic Sign Recognition and Classification

\section{CONCLUSION}

In this paper, many possible studies are discussed so as to provide a clear view of different alternative methods that can be used in the detection, tracking, and grouping of traffic signs. In each of these steps, so many algorithms and methods were applied, and each of these has its own advantages and disadvantages.

The paper also covers the methods of categorization, trends which are used currently and the challenges which are associated with the research of detection and identification of traffic signs and about the ability of the convolutional neural networks to detect the image and the variances in the image with correct accuracy is a really challenging task as it does not give accurate results sometimes. The convolutional neural networks perform the classification and recognition of images correctly even on the damaged and blurred images. It is very likely that for getting better results, more layers should be added in the convolution stage of Neural Network so as to extract more and more features of the images.

In a confusion matrix, classes of highest proportions can be compared to each other and reverse the common factors which could be done with the help of image adjustment. This paper could be a good reference for the researchers who want to know about the current status of the research which is going on in the area of detection and identification of traffic signs. 


\section{ACKNOWLEDGEMENTS}

Our sincere thanks to guide Mrs. Shaili Gupta, Assistant Professor, IMS Engineering College, Ghaziabad, India for her patient guidance and constructive suggestions for the research in the area of traffic sign detection using deep learning. This research is made possible due to her willingness to devout time generously.

\section{REFERENCE}

[1] Yasmina Djebbara, Karima Rebai, Ouahiba Azouaoui, 2018, "Traffic Sign Recognition with Deep Learning”,10.1109/ICASS.2018.8652024.

[2] Kuang Xianyan, Fu Wenbin, Yang Liu, 2018, "Real-Time Detection and Recognition of Road Traffic Signs using MSER and Random Forests", 10.3991/ijoe.v14i03.7925.

[3] Lina-Yung-Huei, Chang-Chen-Chin, Huang Chun-Shu, 2018, "Traffic Sign Detection and Recognition for Driving Assistance System", 10.14738/aivp.63.4603.

[4] Mogelmose Andreas, Trivedi M. Mohan, Moeslund B. Thomas, 2012, "Traffic Sign Detection and Analysis": Recent studies and emerging trends",10.1109/TITS.2012.2209421.

[5] Wali B. Safat, Hannan A. Mahammad, Hussain Aini, Samad A. Salina, 2015, "An Automatic Traffic Sign Detection and Recognition System Based on Colour Segmentation, Shape Matching, and SVM", 10.1155/2015/250461.

[6] Albawi Saad, Mohammed Abed Tareq, Zawi-Al-Saad, 2017, "Understanding of a Convolutional Neural Network”, 10.1109/ICEngTechnol.2017.8308186.

[7] Sinha Aditya, Singh Mradul, Choudhary Nikhil, Rawat Nitin, Vajpayee Pooja, 2020, "Study of a Machine Learning Model for Face Detection, Age Detection, and Gender Recognition", 10.22214/ijraset.2020.5007.

[8] Barathi Anu U. B., Aadesh P.,Balajee R., Balaji M.,2020, " Suspicious Action and Behavior Detection using CNN”,pg. 51-59.

[9] Yamashita Rikiya, Nishio Mizuho, Do Gian Kinh Richard, Togashi Kaori, 2018, “ Convolutional neural networks:an overview and application in radiology",10.1007/s13244018-0639-9.

[10] Hu Wei, Huang Yangyu, Wei Li, Zhang Fan, Li Hengchao, 2015, "Deep Convolutional Neural Networks for Hyperspectral Image Classification", 10.1155/2015/258619.

[11] Benuwa Bright Ben, Zhan Yongzhao Khan, Ghansah Benjamin, Wornyo Keddy Dickson , Kataka Banaseka Frank, 2016, “ A review of Deep Machine Learning", 10.4028/www.scientific.net/JERA.24.124.

[12] Hassaballah M. , Awad Ismail Ali,2020, “ Deep Learning in Computer Vision Principles and Applications", $10.1201 / 9781351003827$.
[13] Wali B. Safat, Abdullah A. Majid, Hannan A. Mahammad, Hussain Aini, Samad A. Salina, Ker J. Pin , Mansor Bin Muhammad, 2019, "Vision Based Traffic Sign Detection and Recognition Systems: Current Trends and Challenges", 10.3390/s19092093.

[14] Xin Mingyuan, Wang Yong, 2019, "Research on image classification model based on deep convolutional neural network", 10.1186/s13640-019-0417-8.

[15] Indolia Sakshi, Goswami Kumar Anil, Mishra S.P.,Asopa Pooja, 2018, "Conceptual Understanding of Convolutional Neural Network-A Deep Learning Approach" ,10.1016/j.procs.2018.05.069. 